\title{
Towards 35\% Efficient Concentrator Modules Based On LPI Köhler Concentrators With External Confinement Cavities
}

\author{
Pablo Zamora ${ }^{1}$, Pablo Benítez ${ }^{1,2}$, Juan Carlos Miñano ${ }^{1,2}$, Yang Li ${ }^{3}$, Marina Buljan ${ }^{1}$ \\ ${ }^{1}$ Cedint, Universidad Politécnica de Madrid, Campus de Montegancedo, 28223 Pozuelo, Spain \\ ${ }^{2}$ LPI-LLC, 2400 Lincoln Ave., Altadena, CA 91001, USA \\ ${ }^{3}$ Zhejiang University, 338 Zheda Road, Hangzhou 310027, China
}

\begin{abstract}
Multijunction solar cells present a certain reflectivity on its surface that lowers its light absorption. This reflectivity produces a loss in electrical efficiency and thus a loss in global energy production for CPV systems. We present here an optical design for recovering this portion of reflected light, and thus leading to a system efficiency increase. This new design is based on an external confinement cavity, an optical element able to redirect the light reflected by the cell towards its surface again. We have proven the excellent performance of these cavities integrated in CPV modules offering outstanding results: 33.2\% module electrical efficiency @ $\mathrm{T}_{\text {cell }}=25^{\circ} \mathrm{C}$ and relative efficiency and $I_{s c}$ gains of over 6\%.
\end{abstract}

Keywords: Concentrator Optics, Confinement Cavity, Nonimaging Optics

PACS: 42.79.Ek Solar collectors and concentrators

\section{INTRODUCTION}

Multijunction cells reflectivity is mainly due to two different reasons: light reflection produced by grid lines and inherent reflectivity of the cell uncovered semiconductor surface (usually covered by an AR coating). Even if reduced to the maximum, these two effects will always be present in any solar cell. Our strategy for improving our CPV module performance is thus based on trying to recover this reflected light in order to increase light absorbed by cell and so global electrical efficiency. This work presents a solution to this problem based on an optical design including an external confinement cavity [1].

The recent invention of high-performance LPI Fresnel-Köhler concentrators (FK [2] and FRXI [3]), fully compatible with the use of these external cavities, have allowed for the practical integration of this structure inside a CPV module [3]. In this way a prototype with cavity has been manufactured, based on a FK (Fresnel-Köhler) architecture. We will present several results for this module: theoretical calculations and experimental (indoors and on-sun) measurements, showing all of them a neat efficiency gain due to the external cavity.

\section{CELL REFLECTIVITY AND CAVITY DESIGN}

High concentration levels produce higher current densities as compared to one-sun situations (no concentration). In the case of multijunction solar cells, the front grid design is optimized for the trade-off between series resistance losses and loss of absorbed light due to front metal grid shading factor $\left(f_{s}\right)$. In today's high concentration multijunction solar cells, $f_{s}$ is in the $8-12 \%$ range. Regarding cell reflectivity, $f_{s}$ and AR coatings are optimized for the $400-900 \mathrm{~nm}$ wavelength range but the reflection in the further infrared is higher (corresponding to top and middle sub-cells range). This does not affect multijunction cells performance because of the bottom sub-cell excess of current.

Two different strategies have been used in the past to minimize this undesired reflection of useful light produced by the front metal grid. The first one consists in preventing the light from hitting the grid lines, for instance using refractive prismatic covers aligned over the grid lines [4]. However, this approach presents several disadvantages. It does not recover the light reflected on the semiconductor surface, needs very precise alignment, and is not effective under wide- 
angle illumination (as occurs in high-concentration systems), especially when a secondary optical element (SOE) is optically coupled to the cell as a secondary concentrator or homogenizer. The second strategy consists on reflective covers, but their implementation also has practical challenges [5].

On the other hand, a third strategy, referred to as external confinement [1], is more compatible with high concentration. It consists on using a refractive cavity with its surface metalized, that collects the light reflected by the cell (either by the grid lines or by the semiconductor surface) and sending it back to the cell. Besides, external confinement does not need precise alignment.

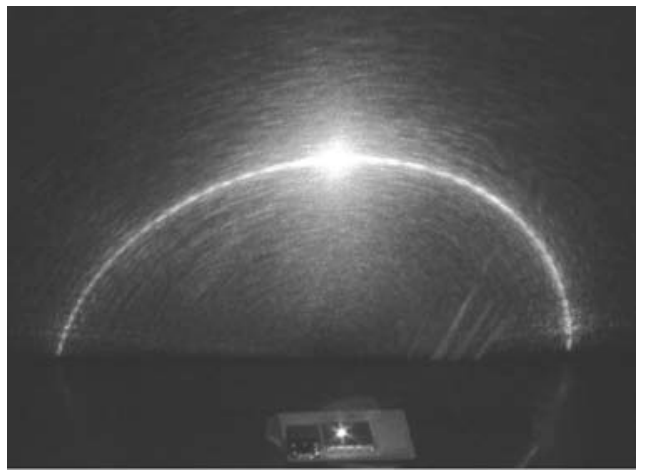

FIGURE 1. Solar cell reflection pattern. Light scattered on the surface of a vertical plane behind the cell. Cell is placed on the bottom part of the figure, with grid lines perpendicular to vertical plane.

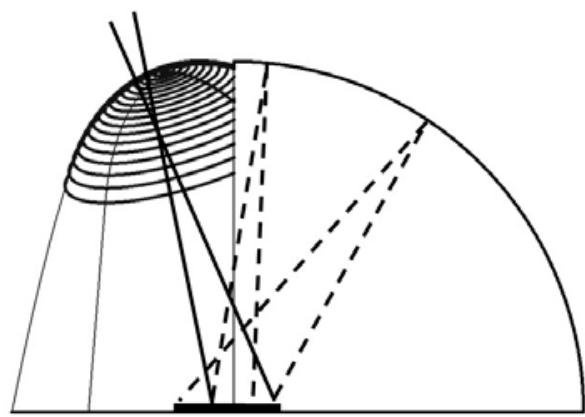

FIGURE 2. Lateral 2D view of FK-Cavity SOE. Refractive part, in the left side; cavity part, in the right. Continuous straight lines mean incident rays; dashed straight lines mean reflected rays. Cell is placed in the bottom part.

For present multijunction cells, the light reflected on the semiconductor surface is specular (the cell surface roughness is very small, similar to good optical mirrors), while the light reflected on the grid lines has a significant diffuse component caused by the grid line geometry and roughness. However, it is remarkable that the roughness and imperfections of the grid line surface still keep the same cylindrical symmetry, as a good approximation. Therefore, the direction of the scattered light conserves the vector component along the grid line, and a light ray is scattered inside the surface of a cone (see FIGURE 1).

This is important for the design of the concentrator and the external cavity (which traditionally has assumed random Lambertian scattering): the illumination of the cell needs to come only be from one hemisphere, while the cavity occupies the other (asymmetric cavity). FIGURE 2 shows this asymmetric cavity concept in the particular case of being applied to an FK concentrator SOE. The figure shows how incident light rays (coming from the module primary element, POE) enter the refractive part of the SOE towards the cell, and the reflected part of this incoming light hits the metalized cavity and returns towards the cell again.

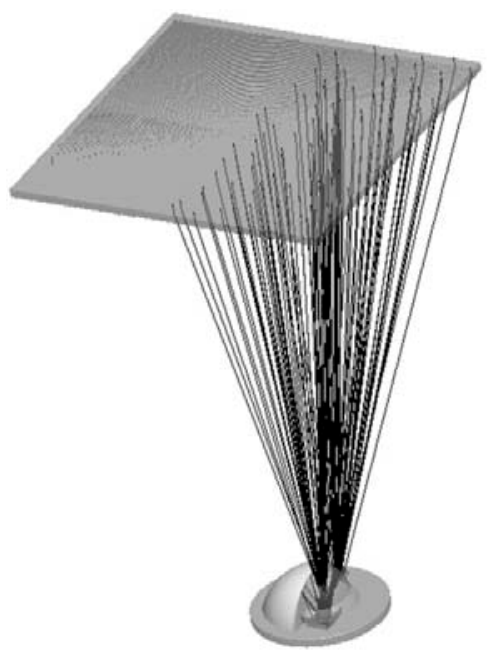

FIGURE 3. FK-Cavity module render with normal incidence ray tracing. Only half of the original FK module POE is used.

As shown in FIGURE 3 rays are focused by primary optical element (a Fresnel lens, like in FK concentrator) on the SOE. Since we are using an asymmetric cavity, for the SOE we are just using two of the original FK four sectors, and the remaining two sectors have been replaced by the cavity. In the same way, to focus light in those two SOE sectors, we just need two of the original POE four-sectors of the FK concentrator.

\section{CAVITY EFFICIENCY GAIN MODEL}

We propose here a mathematical analysis method to model the amount of light recovered by the external cavity (and so the efficiency gain achieved). The recovered portion of light will just depend on two parameters: shading factor $\left(f_{s}\right)$ and concentration $(C)$. Thus we show results calculated for the FK-Cavity 
module (FK with cavity) for different values of $f_{s}$ and $C$ and we compare them with the same calculations made for the FK-NoCavity (original FK concentrator configuration). For this comparison a PMMA POE, a B270 SOE and a $90 \%$ reflectivity external cavity have been chosen for optical concentrators, while for the cell we have used Spectrolab CDO-100-C3MJ efficiency data.
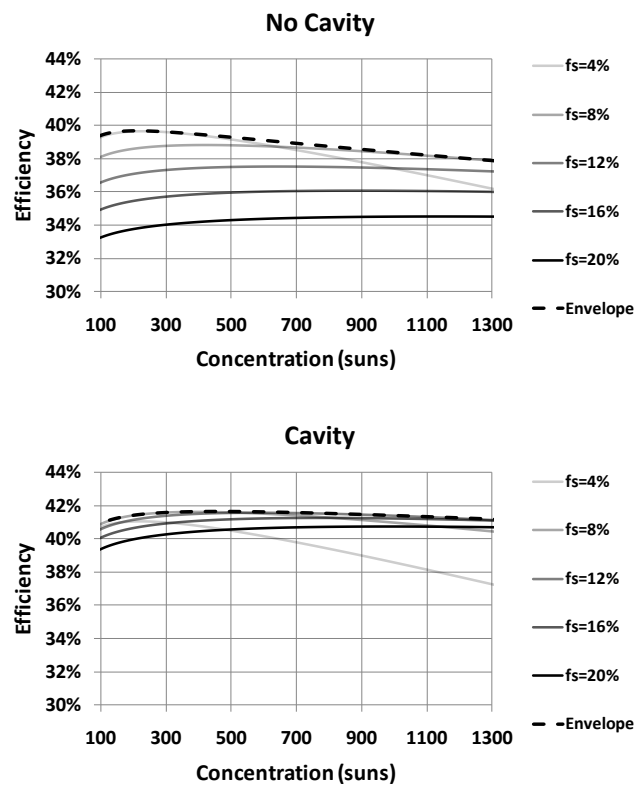

FIGURE 4. Efficiency results for different $f_{s}$ and concentration values for FK-NoCavity (top) and FK-Cavity (bottom). 1 sun $=1 \mathrm{~kW} / \mathrm{m}^{2}$.

These particular results are shown in FIGURE 4, where we offer cell electrical efficiency results with and without cavity (not taking into account optical efficiency). Different curves are plotted for different $f_{s}$ values, except the dashed one, which is the envelope curve of all the other curves (i.e. for each value of concentration it shows the result for the $f_{s}$ value maximizing efficiency). It is simple to see and easy to understand looking at FIGURE 4 that, if we compare a pair of curves with the same $f_{s}$ value, and for a particular $C$, FK-Cavity presents always a higher efficiency than FK-NoCavity. Besides, we can observe that the efficiency gain is higher at higher $f_{s}$ values.

This effect is mainly due to the important loss of absorbed light for FK-NoCavity case, when the cell surface covered by grid lines (i.e. $f_{s}$ ) is large. Moreover, FK-NoCavity presents especially poor efficiency results when dealing with $f_{s}$ values over $10 \%$, for the whole concentration range. However, for the same high $f_{s}$ values, FK-Cavity manages to recover an important part of light reflected by grid lines so efficiency values are outstanding.
Another extracted conclusion for both graphics is that each value of $f_{s}$ has its optimum $C$, and the higher $f_{s}$ is, the higher optimum $C$ value will be [6] (even if this optimum $C$ value is different for both modules). The explanation of this effect has to do with series resistance $\left(R_{s}\right)$. For a given $C, R_{s}$ is lower at higher $f_{s}$ values since we are dealing with higher grid lines area. This situation leads to a trade-off based on $f_{s}$ : low $f_{s}$ values increase semiconductor light absorption, but also increase $R_{s}$, while on the other hand, high $f_{s}$ values decrease semiconductor light absorption, but also decrease $R_{s}$.
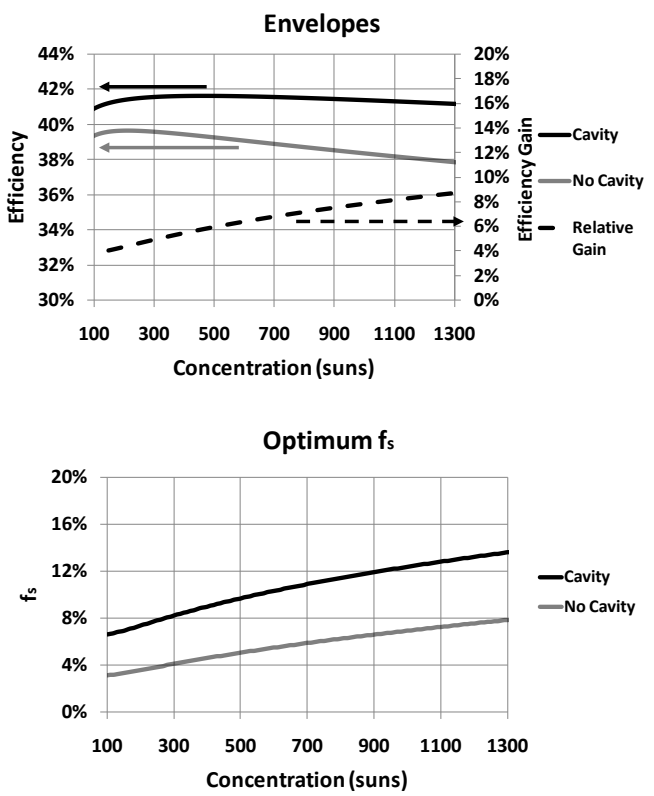

FIGURE 5. Comparison of efficiency results regarding envelope curves (top), and comparison of optimum $f_{s}$ curves in the concentration range (bottom), for FK-Cavity and FKNoCavity.

FIGURE 5 shows in a very neat way the significant efficiency gain introduced by the confinement cavity concept, for any $C$ value. As shown in FIGURE 5 bottom graphic, $f_{s}$ optimum value strongly depends on concentration factor, as stated above. Since high $C$ values mean high optimum $f_{s}$ values, when dealing with high concentrations, FK-NoCavity will lose a great amount of light due to the high density of grid lines. This unabsorbed light will be recovered in the FK-Cavity case thanks to the cavity, so there will be a really significant efficiency difference between both modules when working at high $C$.

\section{MEASURED RESULTS}

This chapter is focused on showing results obtained with our manufactured FK-Cavity module and their 
comparison with the results offered by an equivalent FK-NoCavity module. For this comparative measurements we have used the same housing, POE, solar cell and heatsink for both modules. Thus the only difference between both modules is the SOE (both without AR coating). For this purpose we have used two identical glass pieces (both SOEs with external cavity), metalizing in Ag the cavity for the FK-cavity case (and protecting with paint) and covering cavity with black paint for the FK-NoCavity case (see FIGURE 6). Black paint prevents both effects: reflection due to a metalized surface and Fresnel reflection due to a refractive surface. So we will have a $90 \%$ (approximately) reflectivity cavity in the first case and a $0 \%$ reflectivity in the second one. Our two modules are working at a concentration of 170 suns, and used cell presents a $f_{s}=8 \%$.

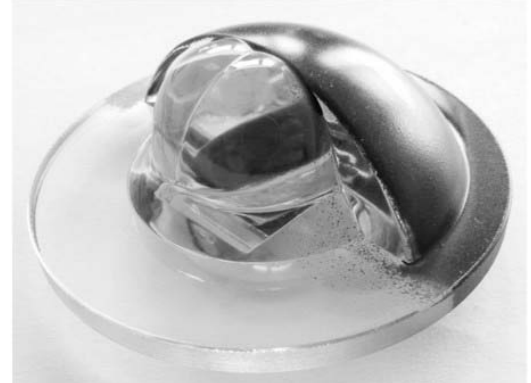

FIGURE 6. Photograph of real SOE with metalized cavity. Paint applied on the cavity surface to protect Ag metallization.

\section{Indoors measurements}

The indoors measurements have been made with a collimated source of white light. We have placed successively our two modules: FK-Cavity and FKNoCavity. In this case we have compared short-circuit currents $\left(I_{s c}\right)$ between both modules, obtaining a very good result: $4.5 \%$ relative gain with the FK-Cavity module.

\section{Outdoors measurements}

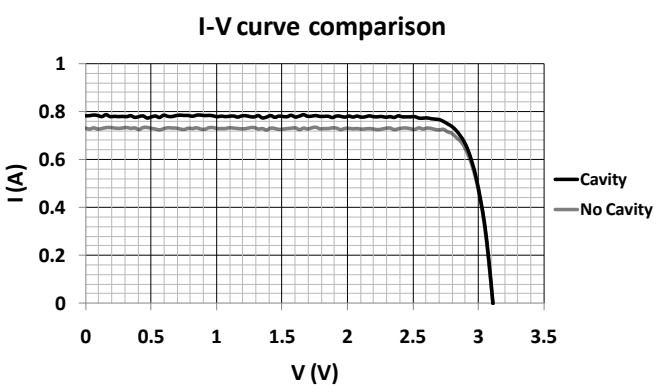

FIGURE 7. FK-Cavity and FK-NoCavity modules compared I-V curves.
To make a fair comparison between both systems, we have measured them at the same moment of the day, so the solar spectrum is exactly the same and so the $J_{\text {ratio }}=J_{\text {top }} / J_{\text {middle }}$ of the solar cell. This second system comparison has offered even better results than the indoors one. As shown in FIGURE 7, for the FKCavity we have a $I_{s c}=0.78 \mathrm{~A}$ and a module electrical efficiency $\eta=32.4 \%$ (33.2\% @ $\left.\mathrm{T}_{\text {cell }}=25^{\circ} \mathrm{C}\right)$, while FKNoCavity presents $I_{s c}=0.732 \mathrm{~A}$ and $\eta=30.6 \%$. This means outstanding results: a $6.0 \%$ electrical efficiency relative gain and a $6.5 \% I_{s c}$ relative gain.

\section{CONCLUSIONS}

Excellent indoors and outdoors results have been obtained with a CPV module including the external cavity concept, which has been successfully proven. This cavity integration in a CPV module has been possible thanks to the use of LPI Fresnel-Köhler advanced concentrator. In this particular case, a FK module has been used for the manufactured prototype.

\section{ACKNOWLEDGMENTS}

Authors thank the Spanish Ministries MCINN (ENGINEERING METAMATERIALS: CSD200800066, DEFFIO: TEC2008-03773, SIGMASOLES: PSS-440000-2009-30), MITYC (ECOLUX: TSI020100-2010-1131, SEM: TSI-020302-2010-65), the Madrid Regional Government (SPIR: 50/2010O.23/12/09,TIC2010 and O-PRO: PIE/209/2010) and UPM (Q090935C59) for the support given in the preparation of the present work. They also want to thank ISI group at the IES (Solar Energy Institute of Madrid), and especially S. Askins, for the help with indoors measurements.

\section{REFERENCES}

1. J. C. Miñano, “Optical confinement in photovoltaics”, in Phisical Limitations to the Photovoltaic Solar Energy Conversion, A. Luque and G.L. Araujo (Bristol,1990).

2. P. Benitez et al. "High performance Fresnel-based photovoltaic concentrator" Opt. Express 18, A25-A40 (2010) .

3. M. Buljan et al., "Improving performances of Fresnel CPV system: Fresnel-RXI Köhler concentrator", 25th EU PVSEC, Valencia (2010).

4. O’Neill, U.S. Patent No. 4,711,972.

5. A. Boca et al., "Prismatic Covers for Boosting the Efficiency of High-Concentration PV Systems," 34 IEEE PVSC (2009).

6. C.Algora and V.Diaz, "Influence of series resistance on guidelines for manufacture of concentrator p-on-n GaAs solar cells”, Prog. in Photovoltaics, 8, 211-225 (2000). 